\title{
Recomendaciones para manejo de pacientes con enfermedad hepática crónica y trasplantados hepáticos durante pandemia por COVID-19
}

\author{
Álvaro Urzúa M. ${ }^{1,2}$, Juan Pablo Arab $V^{3}$, Francisco José Barrera M. ${ }^{3}$, Nidia Fabiola Castro P., \\ Fernando Gómez D. ${ }^{6}$, Katherine González L. ${ }^{2}$, Raúl Lazarte C. ${ }^{1,7}$, Gabriel Mezzano P. ${ }^{4,8}$, \\ Juan Pablo Roblero C. ${ }^{1}$ y Leyla María Nazal O. ${ }^{9}$
}

\footnotetext{
'Sección de

Gastroenterología Departamento de Medicina Interna, Hospital Clínico Universidad de Chile. Santiago, Chile. ${ }^{2}$ Departamento de Gastroenterología Clínica Santa María. Santiago, Chile ${ }^{3}$ Departamento de Gastroenterología, Facultad de Medicina, Pontificia Universidad Católica de Chile. Santiago, Chile. ${ }^{4}$ Sección de

Gastroenterología Clínica Universidad los Andes. Santiago,

${ }^{5}$ Departamento de Gastroenterología, Hospital Clínico de la Fuerza Aérea de Chile.

Santiago, Chile.

${ }^{6}$ Departamento de Gastroenterología

Clínica Alemana.

Santiago, Chile.

Departamento de

Gastroenterología

Clínica Dávila.

Santiago, Chile.

${ }^{8}$ Sección de

Gastroenterología

Hospital del Salvador. Santiago, Chile.

${ }^{9}$ Departamento de Gastroenterología

Clínica Las Condes. Santiago, Chile.
}

Recibido: Aceptado:

Correspondencia a: Dr. Álvaro Urzúa Manchego Aurzuam@hcuch.c
Recommendations for the management of patients with chronic liver disease and liver transplanted recipients during the COVID-19 pandemic

The SARS-CoV-2 virus and the associated disease COVID-19 has quickly become a pandemic. People with underlying chronic diseases or in an immunosupressed state are at risk of having a worse outcome. Cirrhotic patients and liver transplant recipients are considered to be in this higher risk group due to their immunosuppressed state. The aim of this article is to present recommendations based on expert opinion regarding the management of patients with compensated and decompensated liver pathologies who take medication for their immunosuppressed state in medical check-ups and basic treatment management both of patients with and without the COVID-19 disease.

Key words:

\section{Resumen}

El virus SARS-CoV-2 asociado a la enfermedad COVID-19, se han instalado a nivel de pandemia mundial. Las personas portadoras de enfermedades crónicas o estados de inmunosupresión se encuentran en riesgo de desarrollar un curso más grave. Se considera que los pacientes con cirrosis hepática, patología autoinmune o trasplante hepático se encontrarían dentro de este grupo de mayor riesgo por su estado de inmunosupresión. Presentamos recomendaciones de manejo basadas en opinión de experto, en pacientes con patología hepática compensada y descompensada e inmunosuprimidos farmacológicos, en relación a controles médicos y manejo de terapia de base tanto en pacientes sin COVID-19 como en pacientes infectados.

Palabras clave:

\section{Introducción}

El virus SARS-CoV-2 y la enfermedad relacionada a éste, COVID-19, se han instalado ya como pandemia con casos crecientes en nuestro país. Los pacientes con enfermedades crónicas han sido reconocidos como un grupo en que COVID-19 puede tener un curso más grave. No existe actualmente información suficiente de que los pacientes con enfermedades hepáticas crónicas, en particular pacientes con cirrosis (compensada y descompensada) tengan mayor riesgo, sin embargo, el estado de inmunosupresión que acompaña a la cirrosis y sobre todo a la cirrosis descompensada, hacen pensar que sí lo tienen ${ }^{1,2}$. También, en algunas enfermedades hepáticas crónicas que requieren de tratamiento inmunosupresor, como la hepatitis autoinmune, se podría pensar que existe mayor riesgo. Finalmente, los pacientes trasplantados hepáticos debido a su inmunosupresión farmacológica, podrían tener un curso más grave. Esto no ha sido descrito aún en trasplantados, pero sí en otros pacientes inmunosuprimidos (oncológicos $)^{3}$. Además, existe información de que otro tipo de enfermedades virales respiratorias (ej: influenza) pueden tener peor evolución en trasplantados. 
El presente texto no pretende ser una guía terapéutica. Tan sólo corresponde a recomendaciones de expertos en hepatología del directorio de la Asociación Chilena de Hepatología, basados en la escasa literatura acumulada y de su experiencia en manejo de pacientes con hepatopatía aguda, crónica y trasplantados. Se presentan las recomendaciones para distintos grupos de pacientes con enfermedad hepática, además de impacto de SARS-CoV-2 en el hígado.

\section{SARS-CoV-2 e hígado}

Sabemos que SARS-CoV-2 se une a la enzima convertidora de angiotensina II, la cual está presente en colangiocitos y células epiteliales hepáticas ${ }^{4}$. Por esta vía podría generar daño citopático directo. Por otra parte, la respuesta inflamatoria generada por la infección, mediante activación del sistema inmune innato, podría ser responsable de compromiso hepático en ésta infección, tal como ocurre en otras virales ${ }^{5}$. A pesar de lo anterior, hasta ahora el impacto clínico a nivel hepático de la infección no ha sido el más importante.

La alteración del perfil hepático se ha presentado en 14 a $53 \% \%^{1,6}$ de los infectados hospitalizados. Con esta escasa información con la que contamos por ahora se puede decir:

La gran mayoría de los pacientes presentan elevaciones discretas de bilirrubina y de GPT (ALT)/GOT (AST), no mayores a 1-2 veces VN.

a. La gran mayoría de los pacientes presentan elevaciones discretas de bilirrubina y de GPT (ALT)/ GOT (AST), no mayores a $1-2$ veces VN.

b. La ALT elevada se ha asociado a curso más severo de la infección ${ }^{7}$.

c. Pacientes que presenten hepatitis aguda severa o que debutan con hepatitis aguda son anecdóticos hasta ahora ${ }^{8,9}$.

Se desconoce si la enfermedad hepática crónica de cualquier tipo hace más susceptible a la infección por el virus o si éstas se pueden descompensar. Más importante por ahora es el efecto hepatotóxico o la alteración del perfil hepático que puedan generar algunos de los tratamientos utilizados (ej: remdesivir, tocilizumab).

\section{Debido a lo anterior recomendamos:}

a. Buscar otras causas de alteración de perfil hepático en pacientes con infección por SARS-CoV-2 y transaminasas $>$ a 2 veces el VN.

b. Realizar imágenes complementarias sólo si la probabilidad pre-test es elevada, debido a la exposición de los equipos al virus.

c. En pacientes con infección por SARS-CoV-2 en los que se ha iniciado tratamiento específico, siem- pre considerar la hepatotoxicidad por drogas como la causa de alteración del perfil hepático.

d. Realizar control seriado de perfil hepático en pacientes con curso moderado a grave o en los que reciban terapia específica, sobre todo remdesivir y tocilizumab.

e. Reservar la biopsia hepática sólo para pacientes sin etiología clara, con transaminasas $>5-10$ veces el valor normal o aquellos en que su resultado tendrá un impacto importante en la decisión terapéutica (Ej: rechazo celular, hepatitis autoinmune grave).

\section{Pacientes sin COVID-19}

\section{Enfermedad hepática crónica compensada} (incluye cirrosis compensada)

a. Controles médicos:

- Siempre que sea posible, se recomienda el uso de métodos no presenciales, como telemedicina en sus distintos formatos, para control de este grupo.

- Los exámenes de laboratorio pueden ser chequeados por vías no presenciales también.

b. Tamizaje habitual:

- Hepatocarcinoma: El tamizaje habitual cada 6 meses debe idealmente mantenerse, pero podría ser retrasado en 2 meses en casos seleccionados.

- Várices esofago-gástricas: El tamizaje habitual podría retrasarse en grupos de menor riesgo (hepatitis crónica por virus $\mathrm{B}$ con $\mathrm{CV}$ indetectable, hepatitis crónica por virus $\mathrm{C}$ tratada, enfermedad hepática por alcohol en abstinencia). Puede considerarse marcadores no-invasivos de bajo riesgo de presencia de várices esofágicas según Baveno VI (recuento de plaquetas $>150.000 / \mathrm{uL}$ y elastografía $>20 \mathrm{kpa})^{10}$ como aproximación inicial.

c. Tratamiento:

No es recomendable suspensión de ningún tratamiento crónico habitual.

- Hepatitis virales: Mantener tratamiento antiviral habitual.

- Hepatitis autoinmune: referirse a punto 3.

d. Profilaxis:

- La profilaxis primaria de hemorragia digestiva alta variceal (HDAV) debe realizarse según las recomendaciones del consenso de Baveno VI10. Preferir la profilaxis farmacológica en caso de ser posible.

- Énfasis en la importancia de vacunación contra influenza y neumococo.

- Extremar distanciamiento social durante el brote. 


\section{Cirrosis descompensada (incluye pacientes con hepatocarcinoma)}

a. Controles médicos:

- Siempre que sea posible, se recomienda el uso métodos no presenciales, como telemedicina en sus distintos formatos, para control de este grupo. Tener en cuenta que es un grupo de mayor riesgo, por lo que si la información proporcionada no es suficiente o se requiere realizar examen físico, puede ser necesario el control presencial con los elementos de protección personal (EPP) recomendadas por MINSAL ${ }^{11}$ $\mathrm{u}$ otros organismos competentes.

- Los exámenes de laboratorio pueden ser chequeados por vías no presenciales también. Tener en cuenta que puede requerirse un control más estricto.

b. Tamizaje habitual:

- Hepatocarcinoma: El tamizaje habitual cada 6 meses debe idealmente mantenerse, pero podría ser retrasado en 2 meses en casos seleccionados.

- Várices esofago-gástricas: El tamizaje habitual podría retrasarse en grupos de menor riesgo (hepatitis crónica por virus $\mathrm{B}$ con $\mathrm{CV}$ indetectable, hepatitis crónica por virus $\mathrm{C}$ tratada, enfermedad hepática por alcohol en abstinencia). Evaluar realizarse en caso de nueva descompensación.

c. Tratamiento:

- No es recomendable suspensión de ningún tratamiento farmacológico habitual.

- Hepatitis virales: Mantener tratamiento antiviral habitual.

- Hepatocarcinoma:

- Terapias locorregionales (ej: Quimioembolización, terapias ablativas): Intentar no suspender. En casos en que se estime que su retraso en 2-3 meses no impactará el pronóstico, se pueden retrasar.

- Terapias sistémicas: No deben suspenderse.

- Hepatitis autoinmune: referirse a punto 3 .

d. Profilaxis:

- La profilaxis primaria de HDAV debe realizarse según las recomendaciones del consenso de Baveno VI. Preferir la profilaxis farmacológica en caso de ser posible.

- La profilaxis secundaria de HDAV no debería ser suspendida. Para la realización de profilaxis endoscópica, se deben tomar las medidas recomendadas por nuestra sociedad ${ }^{12}$.

- Profilaxis de Peritonitis bacteriana espontánea y encefalopatía hepática: Especial énfasis en su cumplimiento para evitar ingresos por descompensación.
- Énfasis en la importancia de vacunación contra influenza y Streptoccocus pneumoniae.

- Extremar distanciamiento social durante el brote.

3. Hepatitis autoinmune (incluye cirrosis)

a. Para controles médicos, tamizaje y profilaxis referirse a punto 1 o 2 según corresponda.

b. Tratamiento:

- Los pacientes inmunosuprimidos se consideran como grupo de riesgo para COVID-19 grave. No existe información específica en hepatitis autoinmune, sin embargo, la suspensión o disminución de dosis terapéutica, puede desencadenar una descompensación, situación en la que la infección puede ser grave.

- No se recomienda la suspensión ni cambio de tratamiento en pacientes sin COVID-19 ni pacientes contacto de casos COVID-19.

\section{Trasplante hepático}

No hay evidencia aún del rol de la inmunosupresión en infección por SARS-CoV-2; existe incluso información de que es la respuesta inmune innata la responsable del daño pulmonar, por lo que la inmunosupresión podría ser protectora ${ }^{13,14}$. Sin embargo, en la mayoría de las recomendaciones se considera los trasplantados inmunodeprimidos, sobre todo los mayores de 60 años, como un grupo de mayor riesgo de infección severa. La información publicada es aun escasa, pero los pacientes con cuadros más severos corresponden a estos subgrupos ${ }^{15-19}$.

a. Controles médicos:

- Trasplante hace más de 1 año:

- En pacientes con último control sin cambios con respecto a controles previos, se puede retrasar control en 2-3 meses o realizar control mediante mecanismos no presenciales, como telemedicina u otro similar.

- Trasplante hace menos de 1 año:

- Seguir la recomendación de cada equipo de trasplante. En aquellos pacientes que se pueda, recomendamos métodos no presenciales, como telemedicina en sus distintos formatos.

b. Tratamiento:

- No se recomienda la suspensión ni cambio de tratamiento en pacientes sin COVID-19 ni en pacientes contacto de casos COVID-19.

c. Profilaxis

- Recomendamos la vacunación contra influenza en todos los pacientes con un tiempo mayor a 4 meses de trasplante.

- Recomendamos iniciar o completar la vacunación con Streptoccocus pneumoniae en todos los pacientes 
Tabla 1. Recomendaciones para pacientes SIN COVID-19 cirróticos compensados y descompensados, patología autoinmune y trasplante hepático

\begin{tabular}{|c|c|c|c|c|}
\hline & Controles médicos & Tamizaje habitual & Tratamiento & Profilaxis \\
\hline 1. cCALD & Telemedicina & $\begin{array}{l}\text { - HCC: } 6 \mathrm{~m} \text { (puede extender } 2 \mathrm{~m} \text { ) } \\
\text { - VEG: Retrasar } \downarrow \text { riesgo } \\
(\mathrm{FS}<20 \mathrm{kPa} \text {; plaq }>150 \mathrm{~K})\end{array}$ & No suspender & $\begin{array}{l}\cdot 1^{\text {a }} \text { VEG: BBNS } \\
\cdot \theta \text { : influenza y neumococo }\end{array}$ \\
\hline $\begin{array}{l}\text { 2. Cirrosis } \\
\text { descompensada }\end{array}$ & $\begin{array}{l}\text { Telemedicina } \\
\text { (presencial algunos } \\
\text { casos) }\end{array}$ & $\begin{array}{l}\text { - HCC: } 6 \mathrm{~m} \text { (puede extender } 2 \mathrm{~m} \text { ) } \\
\text { - VEG: Retrasar } \downarrow \text { riesgo } \\
\text { (FS }<20 \mathrm{kPa} \text {; plaq }>150 \mathrm{~K}) \\
\text { - Realizar si descomp (¿?) }\end{array}$ & No suspender & $\begin{array}{l}\text { - } 1^{\text {a }} \text { VEG: BBNS } \\
\text { - } 2^{\mathrm{a}} \text { VEG: No susp. } \\
\text { - PBE y EH: intensas } \\
\text { - } 6 \text { : influenza y neumococo }\end{array}$ \\
\hline $\begin{array}{l}\text { 3. Hepatitis } \\
\text { autoinmune }\end{array}$ & $\begin{array}{l}\text { Telemedicina o } \\
\text { preferencial a }\left(\begin{array}{l}1 \\
\text { o }\end{array} 2\right)\end{array}$ & & $\begin{array}{c}\text { No suspender } \\
\text { inmunosupresión }\end{array}$ & Referirse a 1 o 2 \\
\hline $\begin{array}{l}\text { 4. Trasplante } \\
\text { hepático }\end{array}$ & $\begin{array}{l}\cdot<1 \text { año: estable: } \\
\text { telemedicina } \\
\cdot<1 \text { años: caso a caso }\end{array}$ & & & $\begin{array}{l}\text { - influenza (4 m post-THO } \\
\text { y completar neumococo } \\
\text { - Recomendaciones específicas }\end{array}$ \\
\hline
\end{tabular}

HCC: Hepatocarcinoma; VEG: várices esofagogástricas; FS: fibroscan; BBNS: betabloqueadores no selectivos.

d. Información para pacientes

- Recomendaciones según anexo 1 (INSTRUCTIVO COVID-19 PARA PACIENTES TRASPLANTADOS HEPÁTICOS).

Estas recomendaciones se resumen en la Tabla 1.

\section{Pacientes con COVID-19}

\section{Recomendaciones de hospitalización}

a. Enfermedad hepática crónica sin cirrosis

- Sin otras comorbilidades y cuadro leve: Se podría manejar de manera ambulatoria.

- Comorbilidad (HTA, DM2, mayor a 60 años): Inicialmente hospitalizado.

b. Cirrosis (compensada y descompensada), hepatocarcinoma y trasplantados hepáticos: Manejo hospitalizado.

\section{Recomendaciones de tratamiento}

a. Analgésicos y antipiréticos:

- Evitar uso de AINES.

- Preferir uso de Paracetamol, con dosis máxima de 2-3 g (En caso de consumo activo de alcohol no sobrepasar 1,5 g/día).

b. Tratamiento cirrosis descompensada: Siempre que se pueda mantener las terapias específicas para manejo de la hipertensión portal, ascitis, encefalopatía hepática, peritonitis bacteriana espontánea, etc. c. Inmunosupresión:

- Hepatitis autoinmune: En pacientes con curso moderado a grave de infección, se puede considerar disminución o suspensión transitoria de azatioprina y micofenolato, manteniendo tratamiento con prednisona o similar en dosis no menor a $10 \mathrm{mg}$ al día. Discutir con especialista.

- Trasplante hepático: En pacientes con curso moderado a grave de infección, se puede considerar disminución o suspensión transitoria de inmunosupresión. Discutir con especialista.

- Micofenolato y Azatioprina: Suspender o disminuir dosis en casos graves, sobreinfección o linfopenia.

- Inhibidores de calcineurina: Considerar disminuir, pero no suspender, sobretodo en casos graves, sobreinfección o linfopenia.

- Esteroides: No suspender. En caso de reducir dosis, mantener siempre al menos $10 \mathrm{mg}$ al día o equivalente de prednisona.

Estas recomendaciones se resumen en la Tabla 2.

d. Tratamiento específico COVID-19: No existe ninguna evidencia reportada de tratamiento de COVID-19 en los subgrupos de pacientes con hepatopatías crónicas, cirrosis o trasplantados hepáticos. La atención debe estar en los riesgos de hepatotoxicidad e interacción farmacológica. No todos los tratamientos probados hasta ahora para COVID-19 están disponibles en nuestro medio, por lo que nos referiremos a aquellos que sí se encuentran disponibles. 
Tabla 2. Recomendaciones para pacientes con COVID-19 moderado/severo en cirróticos compensados y descompensados, en pacientes con patología autoinmune y en pacientes con trasplante hepático

\begin{tabular}{|c|c|c|c|}
\hline & Prednisona & Azatioprina micofenolato & ICN \\
\hline Hepatitis autoinmune & $\begin{array}{l}\text { - No suspender si usa } \\
\text { - } 10 \text { mg mínimo } \\
\text { - Inicio si suspensión otros }\end{array}$ & Considerar disminuir o suspender & \\
\hline Trasplante hepático & $\begin{array}{l}\text { - No suspender si usa } \\
\text { - } 10 \text { mg mínimo } \\
\text { - Inicio si suspensión otros IS }\end{array}$ & $\begin{array}{l}\text { Considerar disminuir o suspender } \\
\text { - Graves } \\
\text { - Sobreinfección } \\
\text { - Linfopenia }\end{array}$ & $\begin{array}{l}\text { - Disminuir dosis } \\
\text { - Intentar no suspender }\end{array}$ \\
\hline
\end{tabular}

- Lopinavir/Ritonavir: No recomendamos su uso, debido a estudios que han demostrado su baja utilidad. En particular evita su uso es trasplantados debido a interacción con CYP3A4. En caso de utilizarse, se debe disminuir dosis de tacrolimus a $1 / 20-1 / 50$ de dosis habitual No utilizar con mTOR. No utilizar en cirrosis descompensada.

- Cloroquina/Hidroxicloroquina (+/- Azitromicina): No se ha asociado a mayor hepatotoxicidad. Hay escasos reportes de DILI grave. Monitorear ECG basal y luego de seguimiento para evaluar intervalo QT, y considerar estudio de glucosa-6-fosfato deshidrogenasa previo a su uso. Interacciones: inhibidores de calcineurina e inhibidores mTOR.

- Remdesivir: No existen interacciones farmacológicas significativas. Si experiencia en cirrosis, pero se estima que podría ser seguro debido a uso de otros antivirales de la misma clase en hepatitis B y C. Monitorizar transaminasas (elevación de GPT/ALT).

- Tocilizumab: No utilizar en cirrosis descompensada. Puede reactivar VHB. Monitorizar transaminasas (elevación de GPT/ALT).

e. Otros fármacos.

- No recomendamos por ahora la suspensión de IECAs o ARA-II.

\section{Actividad de trasplante hepático durante pandemia}

La utilización de recursos, en particular referido a las unidades de paciente crítico, implicauna dificultad real en la mantención de la actividad de trasplante hepático durante la pandemia. No existe un consenso mundial acerca de cómo debe enfrentarse esta problemática, sobre todo porque el impacto de la infección no ha sido el mismo en las distintas áreas geográficas y aún es un proceso dinámico. Sin embargo, en diversos foros y recomendaciones, hay consenso en intentar mantener la opción de trasplantar a aquellos pacientes más graves, siempre que el beneficio sea mayor y no signifique un riesgo para los pacientes y los equipos de salud ${ }^{1,2}$.

\section{En base a lo anterior recomendamos:}

a. Intentar trasplantar sólo a pacientes en los cuales, la mortalidad a corto plazo sin trasplante es muy elevada (ej: Falla hepática fulminante, MELD elevado, ACLF grado III, hepatitis alcohólica grave, etc.).

b. Evaluar de manera dinámica la disponibilidad de recursos locales, en particular referido a disponibilidad de UPC, pabellón, EPP y hemoderivados.

c. Solicitar PCR de SARS-CoV-2 en donantes, sin importar presencia o no de síntomas de enfermedad.

d. Evaluar presencia de síntomas de COVID-19 en receptores. Independiente de ésto, solicitar PCR de SARS-CoV-2 en todos los receptores.

e. Considerar tener un segundo receptor preparado.

f. Suspender actividad de trasplante de donante vivo, a excepción de trasplante pediátrico de urgencia.

g. Considerar informar a pacientes acerca de la menor probabilidad de trasplante durante la pandemia.

h. Ante casos de difícil decisión, considerar consultar con comités de ética locales.

\section{Otras consideraciones}

\section{Biopsia hepática}

a. Pacientes sin COVID-19

- Diferir en todos los casos que se pueda

- No diferir si existe:

- Sospecha de rechazo celular. 


\section{Artículo Original}

- Elevación significativa de transaminasas (mayor a 5-10 veces) sin causa. Si sospecha alta de hepatitis autoinmune (marcadores autoinmunes muy positivos y estudio negativo de otras etiologías), se podría considerar tratamiento empírico y diferir biopsia. Discutir con especialista.

- Sospecha de neoplasia que se tratará dentro de los próximos meses. b. Pacientes con COVID-19: Diferir en la mayoría. Discutir casos seleccionados.

\section{Procedimientos endoscópicos}

Referirse a guía de recomendación ACHEDSCHGE para el funcionamiento de la unidad de endoscopia durante el brote de Coronavirus (COVID-19) $)^{12}$.

\section{Anexo 1. Instructivo COVID-19 para pacientes trasplantados hepáticos}

El virus SARS-CoV-2 y la enfermedad relacionada a éste, COVID-19, se han instalado ya como pandemia con casos crecientes en nuestro país. Actualmente no existe información en la literatura de que COVID-19 sea más grave en pacientes trasplantados. Sin embargo, sabemos que al estar inmunosuprimidos, toda infección viral puede ser más grave y por lo mismo se deben tomar algunas medidas de prevención.

- Extremar medidas de higiene. El lavado de manos con jabón muy frecuente es muy importante. En ausencia de esta posibilidad, se puede utilizar alcohol gel.

- Evitar contacto directo con personas. En caso de que no se pueda evitar, lavar manos o uso de alcohol gel después.

- Al toser o estornudar, hacerlo sobre el pliegue del codo o utilizar un pañuelo desechable, el cual debe ser eliminado inmediatamente. Lave sus manos o use alcohol gel después de usar el pañuelo.

- No llevarse las manos a la cara.

- Ventilar muy bien los ambientes de la casa y del lugar de trabajo, al menos 2 veces al día.

- Desinfectar bien los objetos que se usan con frecuencia.

- No automedicarse. Ante dudas comunicarse con nosotros o con un médico cercano.

- Evitar contacto con personas enfermas confirmadas o contactos de pacientes con COVID-19.

- Si puede quedarse en su casa sin salir, es lo más recomendable.

- Evitar actividades sociales, sobre todo asistir a lugares donde haya mucha aglomeración de gente.

- Evitar cualquier viaje que no sea estrictamente necesario.

- Mantenerse alejado de sintomáticos respiratorios y de personas en cuarentena o sospechosas.

- Es recomendable la administración de vacuna de influenza si tiene más de 4 meses de trasplantado y de neumococo (se debe repetir la dosis cada 5 años).

- Ante la presencia de fiebre y síntomas respiratorios (tos, dolor de garganta o dificultad respiratoria) en usted o un familiar, contáctese con nosotros o con personal médico cercano, para seguir el protocolo activo en ese momento y evaluar si es necesario realizar estudio para búsqueda de SARS-CoV-2. La dificultad respiratoria es un síntoma grave y debe motivar consulta urgente.

- Acerca del uso de mascarillas, la evidencia es contradictoria. Si la va a usar, la mascarilla quirúrgica es suficiente, no es necesario usar la N95. Lo importante es siempre lavar las manos antes de y después de manipular la mascarilla.

\section{Referencias}

1.- AASLD. Clinical Insights for Hepatology and Liver Transplant providers during the COVID-19 Pandemic. 07 de Abril de 2020. https://www.aasld.org/sites/ default/files/2020-03/AASLD-COVID19ClinicalInsights-3.23.2020-FINAL-v2. pdf.

2.- Boettler T, Newsome PN, Mondelli MU,
Maticic M, Cordero E, Cornberg M, et al. Care of patients with liver disease during the COVID-19 pandemic: EASLESCMID position paper. JHEP Reports (2020). doi: https://doi.org/10.1016/j. jhepr.2020.100113.

3.- Liang W, Guan W, Chen R, Wang W, Li $\mathrm{J}, \mathrm{Xu} \mathrm{K}$, et al. Cancer patients in SARSCoV-2 infection: a nationwide analysis in China. Lancet Oncol. 2020;21:335-7.
4.- Chai X, Hu L, Zhang Y, Han W, $\mathrm{Lu} Z$, Ke A, et al. Specific ACE2 expression in cholangiocytes may cause liver damage after 2019-nCoV infection. BioRxiv 2020 February 4. doi: 10.1101/2020.02.03.931766. [Epub ahead of print].

5.- Fan Z, Chen L, Li J, Tian C, Zhang Y, Huang S, et al. Clinical features of COVID-19 related liver damage. 
MedRxiv 2020 February 28. doi: Evitar actividades sociales, sobretodo asistir a lugares donde haya mucha aglomeración de gente.10.1101/2020.02.26.20026971. [Epub ahead of print].

6.- Guan W-J, Ni Z-Y, Hu Y, Liang W-H, Ou C-Q, He J-X, et al. Clinical characteristics of Coronavirus Disease 2019 in China. N Engl J Med 2020 February 28. doi: 10.1056/ NEJMoa2002032. [Epub ahead of print].

7.- Zhou F, Yu T, Du R, Fan G, Liu Y, Liu Z, et al. Clinical course and risk factors for mortality of adult inpatients with COVID-19 in Wuhan, China: a retrospective cohort study. Lancet. 2020;395:1054-62.

8.- Praneet W, Epstein M, Bernstein D. Covid-19 presenting as Acute Hepatitis. AJG. 2020. [Epub ahead of print].

9.- Chen N, Zhou M, Dong X, Qu J, Gong F, Han Y, et al. Epidemiological and clinical characteristics of 99 cases of 2019 novel coronavirus pneumonia in Wuhan, China: A descriptive study. Lancet 2020;395:507-13.

10.- de Franchis R; Baveno VI Faculty. Expanding consensus in portal hypertension: Report of the Baveno VI Consensus Workshop: Stratifying risk and individualizing care for portal hypertension. J Hepatol. 2015;63:743-52.
11.- MINSAL. Protocolo de uso de equipos de protección personal en la prevención de transmisión COVID-19-SARS-CoV-2 (precauciones adicionales de transmisión por gotitas y contacto).https://www. minsal.cl/wp-content/uploads/2020/03/ PROTOCOLO-DE-USO-DE-EQUIPOSDE-PROTECCIÓN-PERSONALEN-LA-PREVENCIÓN-DETRANSMISIÓN-COVID19-versión-2403-2020-corregido-última-página.pdf.

(Visitado: 5 de abril de 2020).

12.- Guía de recomendación ACHEDSCHGE para el funcionamiento de la unidad de endoscopia durante el brote de Coronavirus (COVID-19).http:// www.sociedadgastro.cl/gastroweb/ documentos/2020/GUIA DE RECOMENDACION_ACHED.pdf. (Visitado: 5 de abril de 2020).

13.- Romanelli A, Mascolo S. Immunosuppression drug-related and clinical manifestation of Coronavirus disease 2019: A therapeutical hypothesis. Am J Transplant. 2020. doi: 10.1111/ ajt.15905. [Epub ahead of print].

14.- D'Antiga L. Coronaviruses and immunosuppressed patients. The facts during the third epidemic. Liver Transpl 2020 March 20. doi: 10.1002/1t.25756. [Epub ahead of print].

15.- Boyarsky BJ, Chiang TP, Werbel WA, Durand CM, Avery RK, Getsin SN, et al.
Early Impact of COVID-19 on Transplant Center Practices and Policies in the United States. Am J Transplant. 2020 Apr 13. doi: 10.1111/ajt.15915. [Epub ahead of print].

16.- Qin J, Wang H, Qin X, Zhang P, Zhu L, Cai J, et al. Perioperative Presentation of COVID-19 Disease in a Liver Transplant Recipient. Hepatology. 2020 Mar 27. doi: 10.1002/hep.31257. [Epub ahead of print].

17.- Bin L, Yangzhong W, Yuanyuan Z, Huibo S, Fanjun Z, Zhishui C. Successful Treatment of Severe COVID-19 Pneumonia in a Liver Transplant Recipient. Am J Transplant. 2020 Apr 3. doi: 10.1111/ajt.15901. [Epub ahead of print].

18.- Bhoori S, Rossi RE, Citterio D, Mazzaferro V. COVID-19 in long-term liver transplant patients: preliminary experience from an Italian transplant centre in Lombardy. Lancet Gastroenterol Hepatol. 2020 Apr 9. pii: S2468 1253(20)30116-3. doi: 10.1016/ S2468-1253(20)30116-3. [Epub ahead of print].

19.- Huang JF, Zheng KI, George J, Gao HN, Wei RN, Yan HD, et al. Fatal outcome in a liver transplant recipient with COVID-19. Am J Transplant. 2020 Apr 10. doi: 10.1111/ajt.15909. [Epub ahead of print]. 